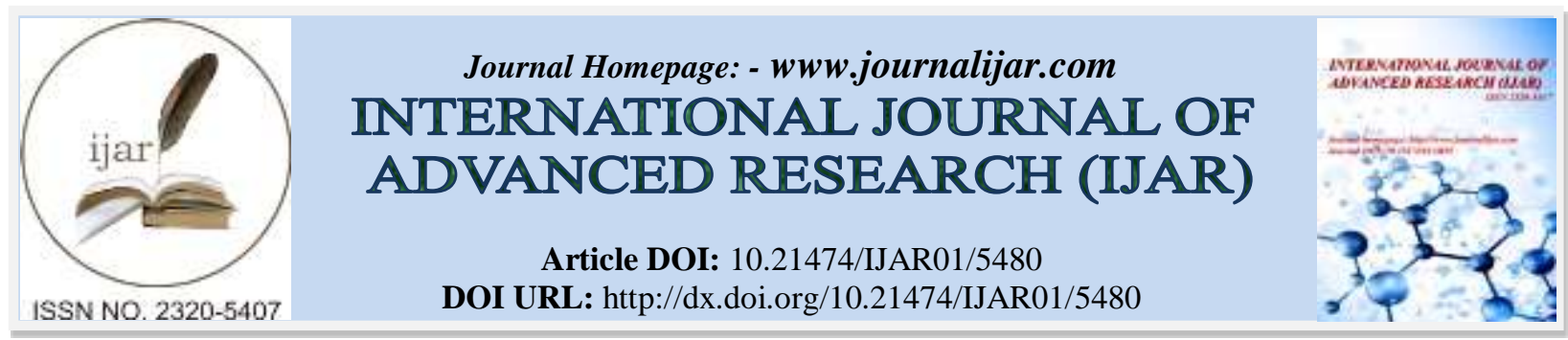

RESEARCH ARTICLE

\title{
BIG FIVE PERSONALITY TRAITS AND ACADEMIC PERFORMANCE OF COLLEGE OF TEACHER EDUCATION STUDENTS AT LAGUNA STATE POLYTECHNIC UNIVERSITY, LOS BAÑOS CAMPUS 2016-2017.
}

Victoria E.Tamban and Ofelia B. Maningas.

Laguna State Polytechnic University, Los Baños Campus, Laguna, Philippines.

\section{Manuscript Info}

Manuscript History

Received: 20 July 2017

Final Accepted: 22 August 2017

Published: September 2017

Key words:-

Big five personality Traits, academic performance, relationships.

\section{Abstract}

This study aimed to determine the relationships of big five personality traits and students' academic performance of College of Teacher Education at Laguna State Polytechnic University Los Baños Campus, Los Baños, Laguna,$^{\text {st }}$ semester Academic Year 2016-2017. The hypothesis stating that no significant relationship between the personality traits and the academic performance of the respondents was determined at ).05 level of significance.

Employing correlational research design. The respondents of the study were the 219 Bachelor of Secondary Education (BSEd) and Bachelor of Elementary Education students (BEEd) from second year, third year and fourth year level. A valid survey questionnaire on determining the level of big five personality traits of the respondents which was adapted from Chowdburry's Individual Level of Big Five Personality Test. Pearson $r$ was used to determine the significant relationship between students' big five personality traits and their' academic performance. Most of the respondents GWA range from 1.50 to 1.74 consist of 70 of $31.96 \%$ while there were 10 or $4.57 \%$ got a GWA of 2.01 above.

The results describe that openness, conscientiousness and extraversion show negative correlation while agreeableness and neuroticism show positive correlation but the relationship between variables are all weak such that the null hypothesis stating that there is no significant relationship between the said variables is rejected with the p-value range from of 0.0001 to 0.0018 which is lower than the level of significance of 0.05. A significant relationship was found between the level of big five personality traits and of students' academic performance.

The results describe that the trait reflects 'open-mindedness' and interest in culture. High scorers tend to be imaginative, creative, and to seek out cultural and educational experiences. Low scorers are more down-toearth, less interested in art and more practical in nature. Most of the students who have taken this test, their score on this dimnsion (25.31) is relatively low.

The students score on conscientiousness (29.68) is about average. High scorers are methodical, well organized and dutiful. Low scorers are less careful, less focused.

Corresponding Author:- Victoria E.Tamban.

Address:- Laguna State Polytechnic University, Los Baños Campus, Laguna, Philippines. 
In extraversion their scores (27.79) is about average wherein high in extraversion are energetic and seek out the company of others. Low scorers (introverts) tend to be more quiet and reserved.

In Agreeableness, student' score on this dimension (29.94) is about average it reflects how they tend to interact with others. People high in agreeableness tend to be trusting, friendly and cooperative. Low scorers tend to be more aggressive and less cooperative.

In Neuroticism (22.40) which is relatively low, this trait reflects the tendency to experience negative thoughts and feelings. High scorers are prone to insecurity and emotional distress. Low scorers tend to be more relaxed, less emotional and less prone to distress.

The researcher concluded that all of the personality traits show significant relationship in the academic.

Based on the conclusions the researchers recommended instructors or teachers must take into consideration thoe students traits as one of the factors affecting students' performance. Seminars on personality development and moral values may be conducted by the College to deepen the students understanding on the importance of having good traits as future educators. Further study is also recommended by the researchers since it is limited only in the College of Teacher Education.

Copy Right, IJAR, 2017,. All rights reserved.

\section{Introduction:-}

Students have individual differences like they differ in their performance in school and they also have different personality traits which affects their academic performance.

As cited in the study of Mohammed Chowdhury students really differ in their personal values; they received information and respond on it differently; their personality trait is different same with their understanding. It is often discussed that a blend of personality characteristics is needed by people to be successful in their field.

There are experts, educators, specialist and researchers have been constantly searching for parsimonious set of variables that predicts patterns of students' behaviors and their relationship to academic achievement. Personality has been recognized as a determining factor on how people learn (Lawrence, 1997; Myer et al, 1998) and according to the findings, all personality traits except extraversion were found to be significantly correlated to students' academic achievement. However, though not statistically significant, there is a positive correlation between extroversion and students' grade, (Chowdburry, M.,2006).

Openness to experience: The individuals scoring high for this trait demonstrate imagination, innovativeness, rule breaking and those who score low tend to act more conventionally and have a conservative outlook. These individuals feel both the good and the bad deeply (Cooper and Miller, 1991), rendering its directional influence on affective reactions like subjective well-being or performance satisfaction unclear. The separation between them and neurotics is that neurotic individuals experience more negative life events than other individuals. While neuroticism entails anxiety and depression (Mount and Barrick, 1995, Costa and McCrae, 1992.

Conscientiousness concerns the way in which we control, regulate, and direct our impulses. Impulses are not inherently bad; occasionally time constraints require a snap decision, and acting on our first impulse can be an effective response. Also, in times of play rather than work, acting spontaneously and impulsively can be fun. Impulsive individuals can be seen by others as colorful, fun-to-be-with, and zany. Nonetheless, acting on impulse can lead to trouble in a number of ways. Some impulses are antisocial. Uncontrolled antisocial acts not only harm other members of society, but also can result in retribution toward the perpetrator of such impulsive acts. Another problem with impulsive acts is that they often produce immediate rewards but undesirable, long-term consequences.

Extraversion is marked by pronounced engagement with the external world. Extraverts enjoy being with people, are full of energy, and often experience positive emotions. They tend to be enthusiastic, action-oriented, individuals who are likely to say "Yes!" or "Let's go!" to opportunities for excitement. In groups they like to talk, assert themselves, 
and draw attention to themselves. Introverts lack the exuberance, energy, and activity levels of extraverts. They tend to be quiet, low-key, deliberate, and disengaged from the social world. Their lack of social involvement should not be interpreted as shyness or depression; the introvert simply needs less stimulation than an extravert and prefers to be alone. The independence and reserve of the introvert is sometimes mistaken as unfriendliness or arrogance. In reality, an introvert who scores high on the agreeableness dimension will not seek others out but will be quite pleasant when approached.

Agreeableness reflects individual differences in concern with cooperation and social harmony. Agreeable individual's value getting along with others. They are therefore considerate, friendly, generous, helpful, and willing to compromise their interests with others'. Agreeable people also have an optimistic view of human nature. They believe people are basically honest, decent, and trustworthy. Disagreeable individuals place self-interest above getting along with others. They are generally unconcerned with others' well-being, and therefore are unlikely to extend themselves for other people. Sometimes their skepticism about others' motives causes them to be suspicious, unfriendly, and uncooperative. Agreeableness is obviously advantageous for attaining and maintaining popularity. Agreeable people are better liked than disagreeable people. On the other hand, agreeableness is not useful in situations that require tough or absolute objective decisions. Disagreeable people can make excellent scientists, critics, or soldiers, (http://www.psychometric-success.com/personality-tests/personality-tests-big-5-aspects.htm)

\section{Neuroticism:-}

The individuals who score high on neuroticism tend to experience effects such as fear, sadness, embarrassment, disgust and anger. Those who score low in this area are usually calm, even-tempered and relaxed at work and in their personal lives. An emotionally intelligent person recognizes and understands the potential consequences of their different emotional states and is able to regulate and control them. Little evidence was found between emotional intelligence and academic intelligence but strong relationships were found between the emotional intelligence dimensions (empathy, autonomy, and emotional control) and the big five, particularly with extraversion and emotional stability (Karen et al, 2002). Schneiderjan et al (2005), however, found strong correlation between emotional stability and academic success in web-based business course. One might argue, in this instance, that students learn better when they are in the company of members of their species other than themselves.

Academic performance shows a satisfactory academic record of the students and they need to meet the obligations of the courses in which they are enrolled but most of the students failed on these obligations due to bulk of requirements and other matters.

\section{Materials and Methods:-}

Correlational research design was employed in this study. The respondents of the study were the 219 selected second year to fourth year students enrolled in the first semester Academic Year 2016-2017 of Laguna State Polytechnic University, Los Baños Campus, Los Baños, Laguna. The sample size of the respondents is computed using Slovin's formula and selected through stratified random sampling.

Researchers' adapted survey questionnaires that serve as an instrument of this study in determining the personality traits of the students from Chowdburry's Individual Level of Big Five Personality Test. Frequency count, percentage, mean, and Pearson $r$ test were used as statistical tools in this study.

This study seeks to describe the respondents' mean level in terms of big five personality traits and to determine the significant relationship between the said variables and the academic performance as shown in figure 1. 


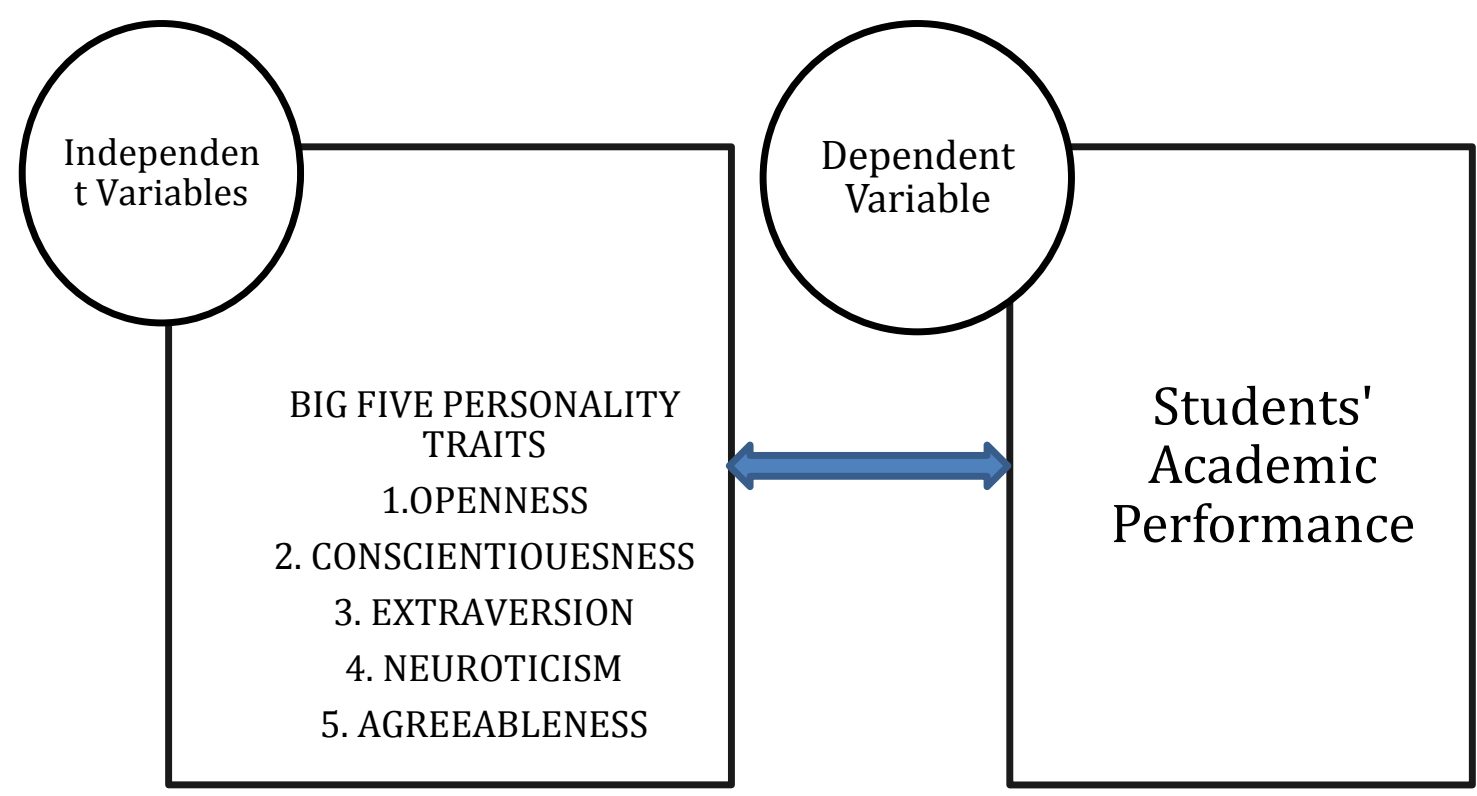

Figure 1:- Research Paradigm

\section{Results and Discussion:-}

The respondents' GWA is illustrated in Table 1.

Table 2:-Student's Academic Performance

\begin{tabular}{|l|l|l|}
\hline GWA & Frequency & Percentage \\
\hline $1.00-1.24$ & 15 & $6.85 \%$ \\
\hline $1.25-1.49$ & 65 & $29.68 \%$ \\
\hline $1.50-1.74$ & 70 & $31.96 \%$ \\
\hline $1.75-2.00$ & 59 & $26.94 \%$ \\
\hline 2.01 -above & 10 & $4.57 \%$ \\
\hline & 219 & $100 \%$ \\
\hline
\end{tabular}

Most of the respondents GWA range from 1.50 to 1.74 consist of 70 of $31.96 \%$ while there were 10 or $4.57 \%$ got a GWA of 2.01 above.

Table 2:-shows the mean level of measurement in terms of students' big five personality traits.

\begin{tabular}{|l|l|l|l|}
\hline Big Five Personality Traits & Mean & SD & Description/Interpretation \\
\hline Openness & 25.31 & 6.60 & Relatively Low \\
\hline Conscientiousness & 29.68 & 2.03 & About Average \\
\hline Extraversion & 27.79 & 9.19 & About Average \\
\hline Agreeableness & 29.94 & 6.95 & About Average \\
\hline Neuroticism & 22.40 & 6.24 & Relatively Low \\
\hline
\end{tabular}

The results describe that the trait reflects 'open-mindedness' and interest in culture. High scorers tend to be imaginative, creative, and to seek out cultural and educational experiences. Low scorers are more down-to-earth, less interested in art and more practical in nature. Most of the students who have taken this test, their score on this dimension (25.31) is relatively low.

The students score on conscientiousness (29.68) is about average. High scorers are methodical, well organized and dutiful. Low scorers are less careful, less focused.

In extraversion their scores (27.79) is about average wherein high in extraversion are energetic and seek out the company of others. Low scorers (introverts) tend to be more quiet and reserved. 
In Agreeableness, student' score on this dimension (29.94) is about average it reflects how they tend to interact with others. People high in agreeableness tend to be trusting, friendly and cooperative. Low scorers tend to be more aggressive and less cooperative.

In Neuroticism (22.40) which is relatively low, this trait reflects the tendency to experience negative thouhts and feelings. High scorers are prone to insecurity and emotional distress. Low scorers tend to be more relaxed, less emotional and less prone to distress.

Table 3:-Analysis on the Relationship between Level of Big Five Personality Traits and Academic Performance

\begin{tabular}{|l|l|l|}
\hline Variables & Computed r-value & p-value \\
\hline Openness \& Academic Performance & -0.016 & $0.0003^{*}$ \\
Conscientiousness \& Academic Performance & -0.0412 & $0.0017^{*}$ \\
Extraversion \& Academic Performance & -0.018 & $0.0003^{*}$ \\
Agreeableness \& Academic Performance & 0.0424 & $0.0018^{*}$ \\
Neuroticism \& Academic Performance & -0.0074 & $0.0001^{*}$ \\
\hline
\end{tabular}

Note. $*=p<.05$

The results describe that openness, conscientiousness and extraversion show negative correlation while agreeableness and neuroticism show positive correlation but the relationship between variables are all weak such that the null hypothesis stating that there is no significant relationship between the said variables is rejected with the p-value range from of 0.0001 to 0.0018 which is lower than the level of significance of 0.05 . A significant relationship was found between the level of big five personality traits and of students' academic performance.

The results of this study revealed that the personality traits show significant relationship in the academic performance of the students which means that personality traits affect their performance in school thus this study recommended instructors or teachers should take into consideration those students traits as one of the factors affecting students' performance. Seminars on personality development and moral values may be conducted by the College to deepen the students understanding on the importance of having good traits as future educators. Further study is also recommended by the researchers since it is limited only in the College of Teacher Education.

Acknowledgements

The authors would like to humbly thank the President of Laguna State Polytechnic University, Dr. Nestor M. de Vera and the very supportive Associate Dean Consorcia S. Tan of College of Teacher Education.

\section{References:-}

1. Ackerman, P.L., Chamorro-Premuzic, T., Furnham, A. (2011). Trait complexes and academic achievement: Old and new ways of examining personality in educational contexts. British Journal Of Educational Psychology, 81(1), 27-40.

2. John V.C. Nye, et.al (2013).Big Five personality traits and Academic Performance in Russian Universities, https://www.hse.ru/data/2013/05/15/1299749297/10PSY2013.pdf

3. http://nobaproject.com/modules/personality-traits

4. https://blog.bufferapp.com/how-the-big-five-personality-traits-can-help-you-build-a-more-effective-team

5. http://www.truity.com/test/big-five-personality-test 